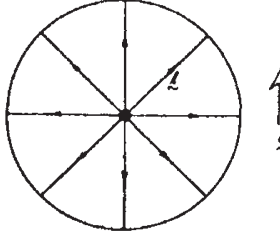

(a)

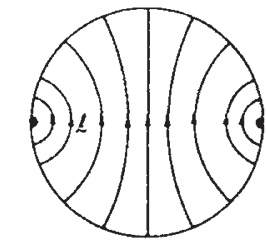

(b)
Fig. 1 Two possible planar textures of the $I$ vector confined in a long cylinder. $a$, Modified de Gennes disgyration with the I vector strictly radial, giving a singularity in the centre, and the d vector uniform. $b$, The 'Pan-Am' texture with two singularities on the walls. $d$ is again uniform (from Gould and Lee, op. cit.).

By a rare coincidence both groups carried out NMR measurements of Aphase textures in the same type of very narrow cylinders ( $2 \mu \mathrm{m}$ diameter). In the experiments an external static magnetic field was applied parallel to the cylinder axes with rf fields parallel and transverse to this to measure the longitudinal and transverse resonances. In addition, Gould and Lee also reported measurements of a transverse resonance with the external magnetic field perpendicular to the cylinders. The confinement of the superfluid in these narrow cylinders has drastic consequences for possible d and I textures. As bending of $\mathbf{I}$ and d usually takes place over a distance of typically $10 \mu \mathrm{m}$, such a deformation will cost quite a lot of energy if it happens over a length of $2 \mu \mathrm{m}$, even more energy than to have a singularity somewhere in the plane of the cylinder. The only known non-singular texture that would fit into a cylinder, the so-called Mermin-Ho vortex, can be shown to require too much bending energy to be a favourable candidate, so that the only possible textures must bear singularities. With a magnetic field parallel to the cylinder one would thus a priori expect planar textures with d uniform across the sample (in the plane of the cylinder cross section) and the l-field looking somewhat as in Fig. 1.

Measuring the transverse NMR signal, both groups find two resonances where initially most of the weight lies in the higher frequency mode; however, this mode slowly decays into the lower mode over $40-60 \mathrm{~min}$, which seems to imply a textural transition. The two groups measured somewhat different NMR frequencies and while the resonances of Gould and Lee proved to be temperature dependent (suggesting a temperature-dependent texture) the one of Saunders et al. did not depend on temperature. Their results for the longitudinal resonances are also different. Gould and Lee find a single, well defined, longitudinal resonance (temperature dependent) which does not change during the textural transi-

\title{
Germs from Mars?
}

\author{
from Arie J. Zuckerman
}

THE health and environmental hazards of bringing to Earth surface soil samples from Mars, assuming that life exists on that planet, have been discussed for over a decade. On the one hand, views have been expressed that the risk of the spread of virulent Martian organisms to terrestrial life forms is so great that such an undertaking planned for the 1980s should be abandoned (see for example Young \& De Vincenzi Science $186,496 ; 1974)$. In a recent cogent discussion of the problem (COSPAR: Life Sciences and Space Research XVI (eds Holmquist \& Strickland) Pergamon, 1978) Martin Favero points out that adequate containment facilities for microorganisms are now available, although absolute security in a scientific sense is of course impossible to guarantee. It is therefore necessary to examine the risks in terms of possibilities.

Favero develops his argument for the unlikelihood of Martian microorganisms' causing harm as follows. If life exists on Mars such organisms would be adapted to low temperature fluctuations and virtually no oxygen or water in the atmosphere. It could reasonably be assumed that such organisms will be so adversely affected hy the Earth's environment that Herculean scientific efforts will be required simply to maintain them. Counter arguments could be that terrestrial microorganisms survive under wide ranges of conditions, which is in principle true, and furthermore Martian organisms might remain unrecognised by the host's immune and other defence mech-

tion, while Saunders et al. do not observe any such resonance, which would be consistent with an axially symmetric texture. Whether the differences in the results can be explained by the different experimental setups (Gould and Lee for example, use Pomeranchuk cooling, Saunders et al. use nuclear demagnetisation) is unclear. The results, however, lead to the following conclusion: in both experiments the first texture seems to decay into a similar one, whose projection into the plane of the cylinder's cross section is about the same (so they give the same longitudinal resonance). The initial textures that would be fairly consistent with the NMR-resonances are the de Gennes disgyration in the case of Saunders et al. and a somewhat distorted 'Pan-Am' texture (Bruinsma \& Maki, private communication) in the anisms thereby resulting in devastating infections. However, in general terms, microorganisms that have no association with man are not pathogenic, for example hydrogen bacteria, methane bacteria and bluegreen algae. Another argument is that extraterrestrial microorganisms might be resistant to inactivation, for instance to heat. It is pointed out that heat which destroys carbon bonds will destroy Martian organisms. If they survived, it would be because their biology was not based on carbon, in which case they should not be pathogenic for forms of life whose biology is based on a carbon system. Finally, there is the potential risk of exchange of genetic material between Martian organisms and terrestrial forms of life. However, there is no evidence of efficient transfer in nature of genetic material between heterogeneous broad groups such as bacteria, algae, fungi, protozoa, higer plants and animals, and the probability of introducing genetic material from Mars to Earth seems insignificant.

This is the stuff of science fiction. Yet similarly conjectural risks had to be considered in the development of atomic energy, production of antibiotics and the use of live virus vaccines.

Arie Zuckerman is Professor and Head of this Department of Medical Microbiology and the WHO Collaborating Centre for Reference and Research on Viral Hepatitis at the London School of Hygiene and Tropical Medicine.

case of Gould and Lee. The deviations of the cylinder cross sections from a circular shape-they have a rounded hexagonal structure as can be seen on magnifications-probably need to be considered. The only alternative mechanism that could, at least qualitatively, account for a distortion of textures in the direction of the cylinder axes in both experiments and the temperature dependence in Gould and Lee's results seem to be superfluid currents in the cylinders.

To explain quantitatively what textures are responsible for the measured NMR resonances will not be an easy but most certainly a very rewarding task. It will provide us with new understanding of textures and singularities in superfluid ${ }^{3} \mathrm{He}$, and beyond that in condensed matter systems. 